\title{
Analysis of the Asymmetric Gene Expression between the Left and Right Hemispheres of Drosophila Brain
}

\author{
Won-Ju Chung \\ Department of Neuroscience, Krieger School of Arts and Sciences, Johns Hopkins University, Baltimore, \\ MD, USA \\ Email: wchung12@jhu.edu
}

Received 11 August 2015; accepted 11 September 2015; published 15 September 2015

Copyright @ 2015 by author and Scientific Research Publishing Inc.

This work is licensed under the Creative Commons Attribution International License (CC BY). http://creativecommons.org/licenses/by/4.0/

(c) (i) Open Access

\section{Abstract}

Studying the molecular mechanism of brain asymmetry can provide important clues to understand neurological diseases and psychiatric disorders related to brain lateralization. In this paper, asymmetric gene expression in the left/right hemispheres of Drosophila brain was genome-widely analyzed to help understand the molecular mechanism of brain asymmetry. Using microarray analysis of total RNAs of the left/right brain hemispheres, thirty-eight genes were found to be differentially expressed in the left/right hemispheres. This result supports that Drosophila brain is asymmetrical at the molecular level. Among thirty-eight genes, six genes of interests were chosen for further analysis based on their protein structures or previous studies: dpr6, CG13299, CG13068, Lim 3, CG43759, and Ir21a. Those six genes encode proteins that serve various functions like neural gene expression, memory control, ion channel, and membrane receptor. Surprisingly, all six genes of interests have their peak expression during the early embryonic stages, suggesting that they may play a role in the developmental stage of brain lateralization. Overall, these findings of differential gene expressions in the left/right brain hemispheres can serve as a basic foundation for further research on the understanding of the molecular mechanism of brain asymmetry.

\section{Keywords}

Brain Lateralization, Gene Expression, Transcriptome Analysis

\section{Introduction}

Striking internal asymmetries of outwardly symmetrical organisms have long been a fascinating subject in the

How to cite this paper: Chung, W. (2015) Analysis of the Asymmetric Gene Expression between the Left and Right Hemispheres of Drosophila Brain. Journal of Behavioral and Brain Science, 5, 440-447. 
field of biology ever since the discovery of the unilateral Nodal signaling [1]. Brain is one representative body part of which the asymmetrical structure is critical for its functions [2]. Based on numerous case studies and experiments, the study of structural asymmetries in the brain has been considered very helpful to understand lateralized brain functions. For instance, the left-hemispheric specialization for speech could be fully studied and substantiated by the discovery of structural asymmetry between the left/right plana temporale [3], which is significantly larger and developed on the left side and spatially coinciding with a brain region that subserve speech [4].

Functional specialization of the human brain hemispheres is apparent from the patients with aphasia caused by the paralysis of the right side, but not left, of their body [5]. Brain laterality is often characterized by the broad generalization that the left hemisphere is responsible for logical processes, whereas the right hemisphere is responsible for creativity and emotion. Indeed, two brain hemispheres are known to have distinctive functional differences not only in humans, but also over a range of vertebrate and invertebrate species [6]. Furthermore, disturbances in brain lateralization often strike in patients with neurological illnesses like autism [7], schizophrenia [8], dyslexia [9], and stuttering [10]. Therefore, profound understanding in the molecular mechanism of brain asymmetry can provide important clues to understand neurological diseases and psychiatric disorders related to brain lateralization.

Numerous researches have been done to find the relationship between brain asymmetry and human behaviors including speech and language, movement and sensation, and handedness. Although functional lateralization of human brain can be roughly pictured by neuroimaging, it is extremely difficult to conduct controlled experiment to elucidate the molecular and cellular processes involved in brain asymmetry. One research analyzed the differential gene expression in the left/right human brain hemispheres by using global transcriptome analysis technique, and the result suggested that those differentially expressed genes might be evolutionarily or developmentally involved in brain lateralization [11]. As such, molecular biology-based research may provide new opportunities in understanding the molecular mechanism of brain asymmetry.

This paper seeks to provide a foundational study on the molecular difference in the left/right brain hemispheres in the simpler and genetically manipulative animal model. One of many difficulties in the research of brain asymmetry is the practical challenge in conducting experiment with human brain. Human brain is not only limited in resource but also extremely complex in both structure and function. To overcome such practical difficulties of studying human brain, Drosophila melanogaster was used in this research as a realistic model for understanding the molecular mechanism of brain asymmetry. Drosophila has two brain hemispheres, though primitive, which exhibit significant structural differences between each other [12]. Although the study makes a brief point about the difference between left and right brain hemisphere in the molecular level, there are no follow-up studies to further explain the molecular mechanism of Drosophila brain asymmetry. In this investigation, therefore, asymmetric gene expression in the left/right hemispheres of Drosophila brain was analyzed at the molecular level to provide clues to understand the molecular mechanism of brain asymmetry.

\section{Materials and Methods}

\subsection{Fly Stocks}

The female $w^{1118}$ fly was used in the experiments. The flies were grown on standard cornmeal-yeast-agar medium at $25^{\circ} \mathrm{C}$.

\subsection{Microdissection of Drosophila Brain}

Using a sharp needle, the head was cut off from Drosophila body. Then, the head was bilaterally dissected into two parts, each consisting of the left and right hemispheres of Drosophila brain. To prevent the denaturation of brain mRNA, dissected brain hemispheres were immediately separated and stored in dry ice to freeze. As a result, 250 of left and right brain hemisphere were collected. The same procedure was repeated to make two samples of the left (L-1 and L-2) and right (R-1 and R-2) brain hemispheres.

\subsection{RNA Extraction and Microarray Analyses}

For microarray analyses, total RNA was extracted from four samples, each consisting of 250 left or right brain hemisphere of $w^{1118}$ flies, using RNeasy Mini Kit column (Qiagen, Germany). Microarray analyses were per- 
formed with Affymetrix Drosophila Genome 2.0 Array according to the manufacture's procedure (http://www.affymetrix.com), with help of the genome research facility, Seoul National University, Korea. The data were analyzed by using GoMiner program.

\subsection{Quantitative Reverse Transcription PCR Analysis}

RNA (3 $\mu \mathrm{g}$ ) was transcribed reversely by M-MLV reverse transcriptase (Promega). For quantitative real time PCR, the synthesized cDNA was mixed with SYBR Green (Enzynomics, South Korea, \#RT500) and appropriate primers (300 $\mathrm{nM}$ ) and then applied to Bio-Rad iQ5 Real-time PCR detection system. The primers that were used to amplify brain cDNA are listed in Table 1.

\subsection{Statistical Analysis}

Student's unpaired $t$-test was used for statistical comparison using Graphpad Prism 5. $p<0.05$ was considered statistically significant.

\section{Results}

\subsection{Preparation of Total RNA from the Left/Right Drosophila Brain Hemispheres}

Female $w^{1118}$ Drosophila melanogaster was used for this investigation. The head was first cut off from Drosophila body and then bilaterally dissected into two parts, each consisting of the left and right brain hemispheres. As a result, 250 of each brain hemisphere were collected. For further analyses, total RNA was extracted from two samples of each brain hemisphere as described in Materials and Methods. The experiment was repeated from collecting the same number of brain hemispheres.

\subsection{Global Transcriptome Analysis between the Left/Right Hemispheres}

The gene expression of the left/right brain hemisphere sample groups was analyzed by microarray analysis technique. Two left hemisphere samples (L-1 and L-2) and two right hemisphere samples (R-1 and R-2) were analyzed and compared to identify the genes with significantly different expression level.

In the experiment design, the left hemisphere was set up as the comparison group and the right as the experimental group. Based on Student T-test (p-value $<0.05$ ) and fold change (cutoff $=1.5$ ), genes with significantly different left/right expression level were identified in the volcano plot (Figure 1(a)). Interestingly, twenty-five genes were significantly more expressed in the right hemisphere compared to in the left hemisphere; on the other hand, thirteen genes were significantly less expressed in the right hemisphere than in the left hemisphere.

\begin{tabular}{ccc}
\hline Table 1. PCR primer list. & & \\
\hline Name & Sequence & Tm $\left({ }^{\circ} \mathrm{C}\right)$ \\
\hline Lim3-5 & TCGACTTCAGCAACATGAGC & 58.54 \\
Lim3-3 & GGCCTAACCAATGTTGTGCT & 58.50 \\
Ir21a-5 & TTGGTATGTCCGGTATATATATAAC & 52.33 \\
Ir21a-3 & TTTTCTTACTCAGGGTTCCAA & 55.06 \\
CG13068-5 & TTCAAGCTGGTGAGTTGGTG & 58.28 \\
CG13068-3 & CCACGTACTGCGAACTGGTA & 58.24 \\
$d p r 6-5$ & GTGTTCAGGGACCCATAGAG & 55.44 \\
$d p r 6-3$ & CACACGGCAGCTGAGATA & 55.15 \\
CG13299-5 & CTCAGAAAGCAAGATGGTTTT & 54.80 \\
CG13299-3 & TATGAAGGTCCTGCCAAAGT & 55.76 \\
CG43759-5 & GACCATCCGGCTGCTACTAC & 58.21 \\
CG43759-3 & GACGGGTAAAGTGCGAGGAA & 60.96 \\
\hline
\end{tabular}




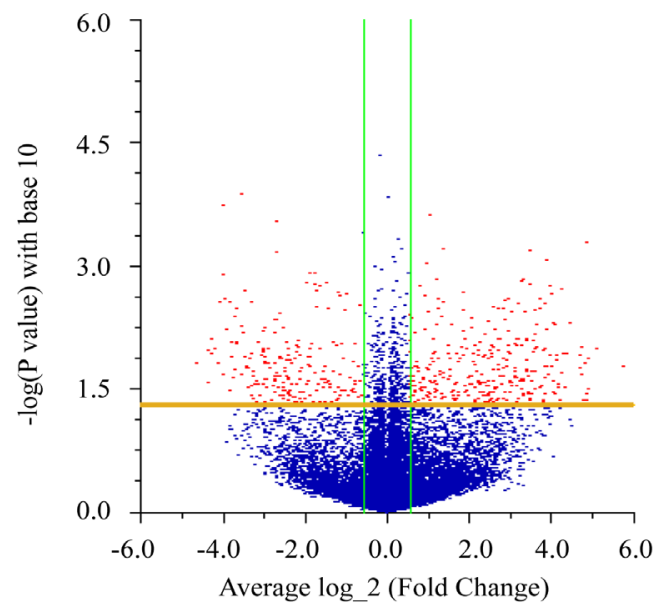

(a)

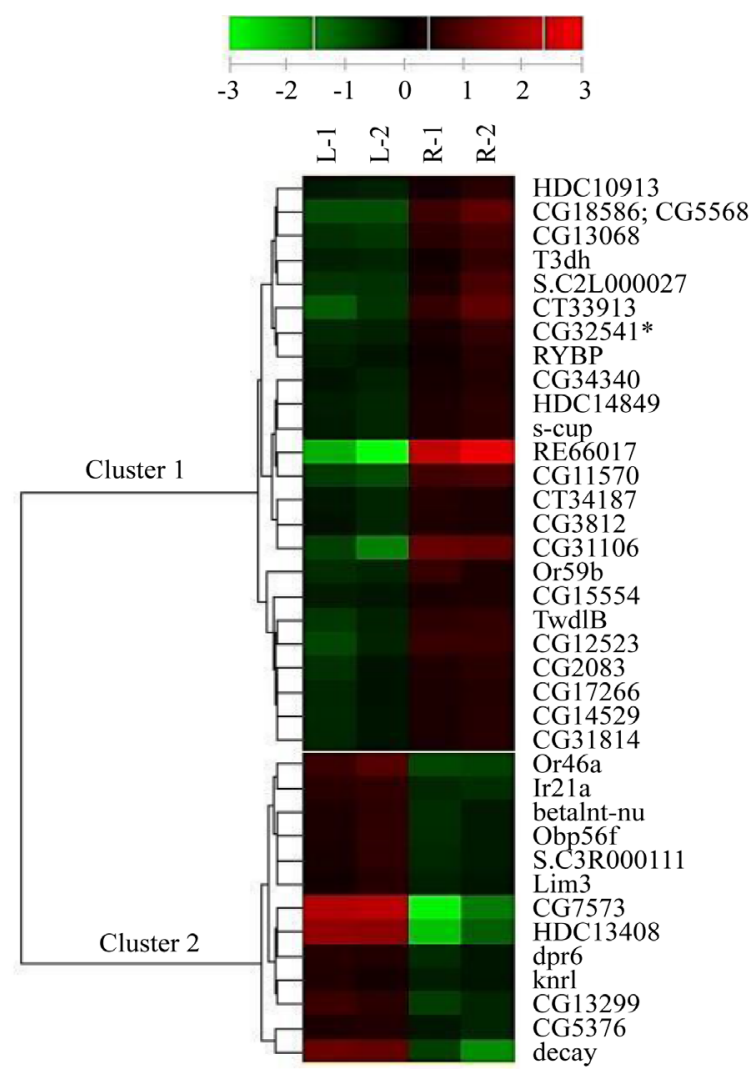

(b)

Figure 1. Microarray analyses. (a) Volcano plot. Based on Student T-test (p-value $<0.05$ ) and fold change (cutoff $=1.3$ ), significant genes are shown in the volcano plot. Red points in the upper left side represent the genes of p-value $<0.05$ among genes of down regulation, and the red points in the upper right side represent the genes of p-value $<0.05$ among genes of up regulation. As a result, twenty-five genes were significantly more expressed in the right brain hemisphere than in the left, and thirteen genes were significantly more expressed in the left brain hemisphere than in the right. (b) Hierarchical clustering of heat maps. The heat maps generated from the microarray analyses of the left/right Drosophila hemisphere samples were subject to hierarchical clustering analysis. Relative expression patterns of the significant genes in left/right hemispheres were shown by the strength of red and green colors, each representing high (Cluster 1) and low (Cluster 2) expression level. Twenty-five genes in Cluster 1 are shown: CG18586, CG5568, CG13068, T3dh, CG43759* (former name: CG32541), RYBP, CG34340, s-cup, CG11570, CG3812, CG31106, Or59b, CG15554, TwdlB, CG12523, CG2083, CG17266, CG14529, CG31814, RE66017, HDC10913, S.C2L000027, HDC14849, CT33913, and CT34187. Thirteen genes in Cluster 2 are shown: Or46a, Ir21a, betaInt-nu, Obp56f, Lim3, CG7573, dpr6, knrl, CG13299, CG5376, decay, S.C3R000111, and HDC13408.

The heat maps generated from the microarray analyses of the left/right Drosophila hemisphere samples were subject to hierarchical clustering analysis (Figure 1(b)). The hierarchical clustering heat map demonstrated that thirty-eight genes were differentially expressed in the left/right brain hemispheres. By using hierarchical clustering technique, relative expression patterns of the significant genes in left/right hemispheres were shown by the strength of red and green colors, each representing high and low expression level. As a result, 25 genes were significantly more expressed in the right brain hemisphere than in the left (CG18586, CG5568, CG13068, T3dh, CG43759, RYBP, CG34340, s-cup, CG11570, CG3812, CG31106, Or59b, CG15554, TwdlB, CG12523, CG2083, CG17266, CG14529, CG31814, RE66017, HDC10913, S.C2L000027, HDC14849, CT33913, and CT34187). On the other hand, 13 genes were significantly more expressed in the left brain hemisphere than in the right (Or46a, Ir21a, betaInt-nu, Obp56f, Lim3, CG7573, dpr6, knrl, CG13299, CG5376, decay, S.C3R000111, and $H D C 13408)$.

The thirty-eight significant genes were then categorized by three domains of gene ontology-biological process, cellular component, and molecular function (Figure 2). In addition, the KEGG pathway analysis was conducted among them. As a result, the significant genes were involved in many different biological processes, such as organ development, sensory perception of chemical stimulus, cell adhesion, regulation of transcription, nervous 


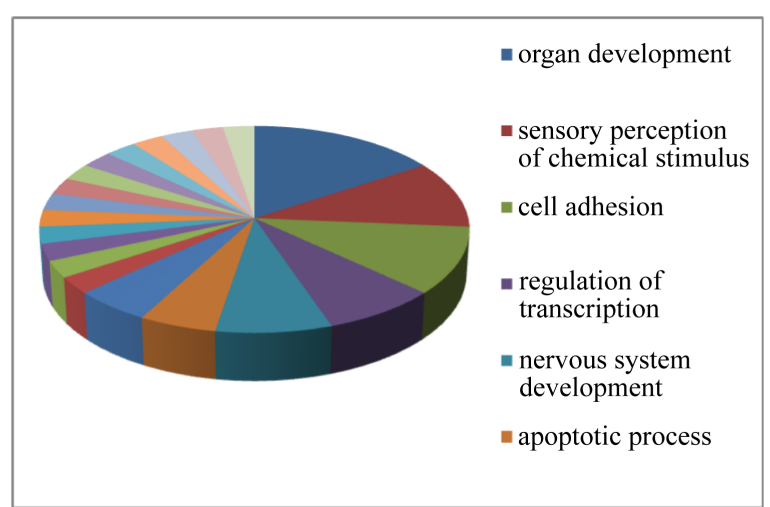

(a)

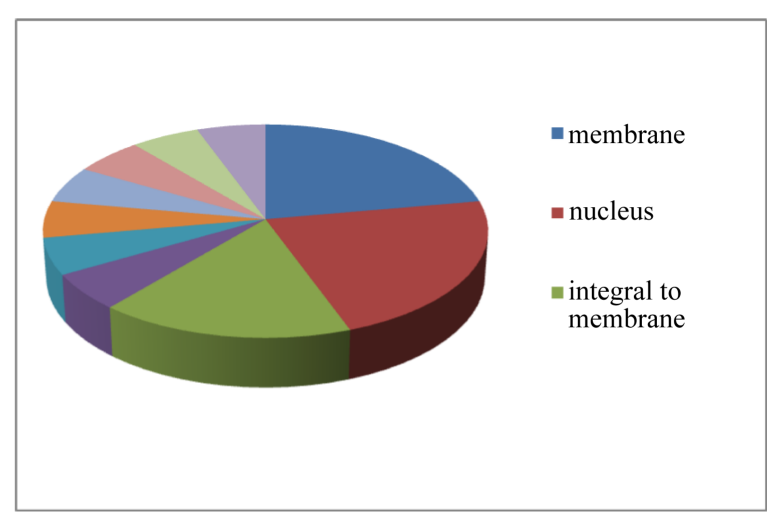

(b)

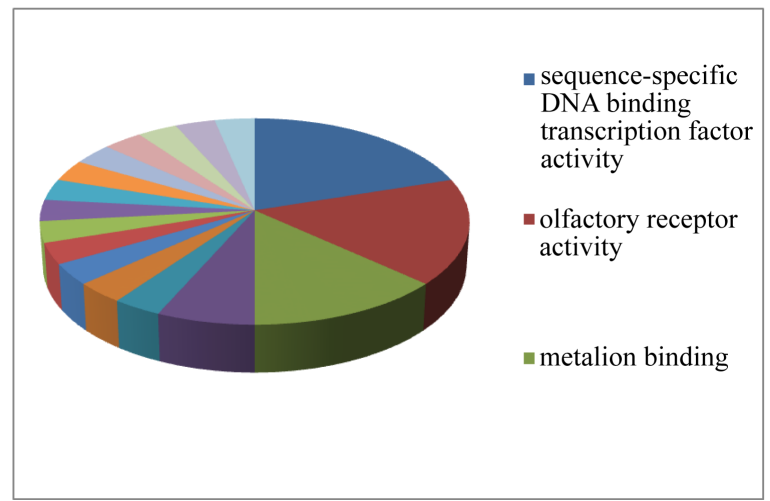

(c)

Figure 2. Gene ontologies of the significant genes represented by pie charts. (a) Genes were categorized by biological process. Significant genes were involved in various biological processes, including organ development, sensory perception of chemical stimulus, cell adhesion, regulation of transcription, nervous system development, and apoptotic process. (b) Genes were categorized by the location in cellular component. Vast majority of the significant genes were found in the membrane or the nucleus. (c) Genes were categorized by their molecular function. Significant genes were responsible for many different molecular functions, including sequence-specific DNA binding transcription factor activity, olfactory receptor activity, and metal ion binding.

system development, and apoptotic process (Figure 2(a)). In terms of cellular component, vast majorities of the significant genes were found in the membrane and the nucleus (Figure 2(b)). Lastly, significant genes were found to serve various molecular functions, including sequence-specific DNA binding transcription factor activity, olfactory receptor activity, and metal ion binding (Figure 2(c)).

\subsection{Reverse Transcription PCR}

Among thirty-eight genes that were significantly differently expressed in the left/right brain hemispheres, six genes of interests were chosen for further analysis: dpr6, CG13299, CG13068, Lim3, CG43759, and Ir21a. To confirm the results from microarray analysis, RT-PCR was used. Significant differences in gene expression between the left and right brain hemispheres were validated by RT-PCR technique. As a result, all six genes were confirmed to have differential gene expression in left/right brain hemispheres (Figure 3). dpr6, CG13299, Lim3, and Ir21a were significantly more expressed in the left brain hemisphere, and CG13068 and CG43759 were significantly more expressed in the right brain hemisphere.

\section{Discussion}

This genome-wide study sought to provide a foundational work on the differential gene expression of the left/ right brain hemispheres of Drosophila. Surprisingly, thirty-eight genes were found to be expressed differentially in the left/right brain hemispheres with statistical significance. Among all significant genes, six genes of interest 
$\operatorname{Lim} 3$

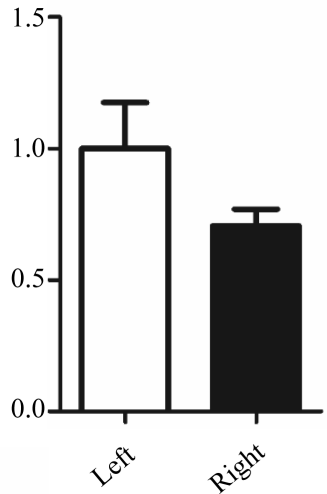

$\operatorname{cg} 13299$

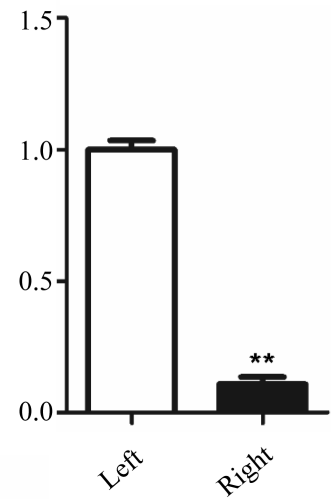

IR21a

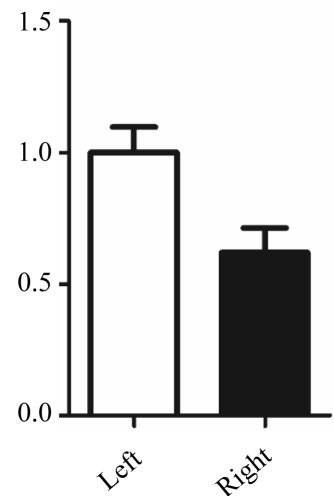

$\operatorname{cg} 43759$

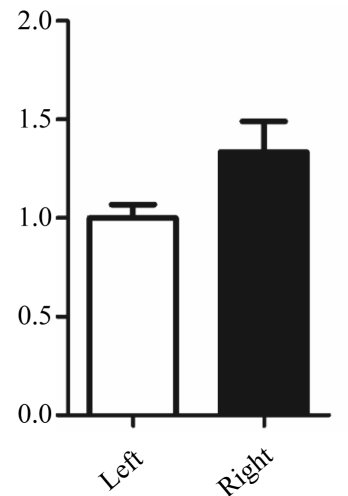

dpr6

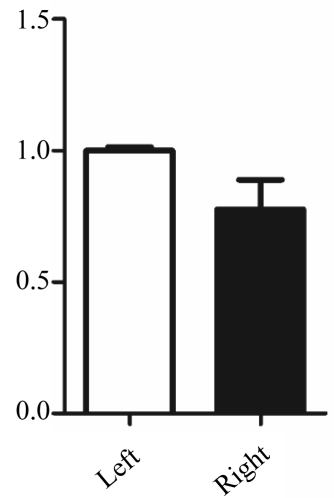

$\operatorname{cg} 13068$

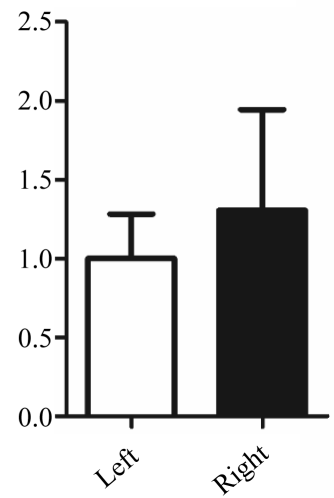

Figure 3. Gene expression levels of six genes of interest in the left/right Drosophila brain hemispheres. Results from RT-PCR as described in Materials and Methods. The tested genes are listed in the order of Lim3, Ir21a, dpr6, CG13299, CG32541, CG43759, and $C G 13068$. The values in $\mathrm{Y}$ axis represent fold changes of gene expression. Data are presented as mean $\pm \operatorname{SEM}(* * p<0.01)$.

were chosen for further analyses based on the previous studies or their protein structures. Those six genes encode proteins that serve various functions like neural gene expression, memory control, ion channel, and membrane receptor, suggesting their relation to the development and lateralized functions of the central nervous system.

The gene Lim3 encodes a protein that is located in the nucleus, containing two cysteine-rich LIM zinc-binding domains and a homeobox DNA-binding domain [13]-[15]. Lim3 functions as sequence-specific DNA binding transcription factor and is involved in various biological processes, including nervous system development [13], motor neuron axon guidance [14], and regulation of cell cycle [15]. The gene expression is peaked in the embryonic stage and early larval stages. Its human homologue is LIM homeobox 3, a member of a large protein family that carries the LIM domain. It is required for pituitary development and motor neuron specification, and its mutations can cause combined pituitary hormone deficiency [16].

Two of the selected genes encode transmembrane receptor proteins. The gene Ir21a (ionotropic receptor 21a) encodes a structure related to extracellular glutamate-gated ion channels and its gene expression is peaked in the embryonic stage. The gene dpr6 (defective proboscis response 6) encodes a transmembrane protein that carries immunoglobulin domains. Inferred from sequence and structural similarity with $d p r 1, d p r 6$ is predicted to be involved in sensory perception of chemical stimulus [17]. Its gene expression is peaked during the very early embryonic stages, and little or no expression is detected in any larval or adult organs/tissues. Such a gene expression pattern suggests that $d p r 6$ is very likely to be involved in the early neuronal development. 
Other genes regulate many functions related to various neural activities. CG13299 showed the most dramatic difference of gene expression level in the left/right brain hemispheres (Figure 3). A study found that the mutant (Toi) near 3' of CG13299 showed defects in one-day memory, hinting the gene's role in the memory control [18]. CG43759 (former name: CG32541) encodes a protein that is involved in the inter-male aggressive behavior [19]. CG13068 is highly expressed in the embryonic stages, during early larval stages, and at stages throughout the pupal period. It is down-regulated in trx mutants [20].

Many of these genes are not yet studied in depth, but further studies of their functions in the lateralized brain can lead us to the better understanding of the molecular mechanism of brain lateralization. Even if those genes are not directly linked to the formation of brain asymmetry, understanding how their gene expressions are controlled will provide us with the critical clues of the molecular network that regulate brain lateralization. Therefore, these findings of differential gene expressions in the left/right brain hemispheres can serve as a basic foundation for further research on the understanding of the molecular mechanism of brain asymmetry.

\section{References}

[1] Levin, M., Johnson, R.L., Stern, C.D., Kuehn, M. and Tabin, C. (1995) A Molecular Pathway Determining Left-Right Asymmetry in Chick Embryogenesis. Cell, 82, 803-814. http://dx.doi.org/10.1016/0092-8674(95)90477-8

[2] Watkins, K.E., Paus, T., Lerch, J.P., Zijdenbos, A., Collins, D.L., Neelin, P., Taylor, J., Worsley, K.J. and Evans, A.C. (2001) Structural Asymmetries in the Human Brain: A Voxel-Based Statistical Analysis of 142 MRI Scans. Cerebral Cortex, 11, 868-877. http://dx.doi.org/10.1093/cercor/11.9.868

[3] Broca, P. (1861) Nouvelle observation d’aphémieproduite par unelésion de la troisièmecirconvolutionfrontale. Bulletins de la Sociétéd'anatomie (Paris), 2e serie, 6, 398-407.

[4] Geschwind, N. and Levitsky, W. (1968) Human Brain: Left-Right Asymmetries in Temporal Speech Region. Science, 161, 186-187. http://dx.doi.org/10.1126/science.161.3837.186

[5] Cubelli, R. and Montagna, C.G. (1994) A Reappraisal of the Controversy of Dax and Broca. Journal of the History of the Neurosciences: Basic and Clinical Perspectives, 3, 215-226. http://dx.doi.org/10.1080/09647049409525614

[6] Rogers, L.J., Vallortigara, G. and Andrew, R.J. (2013) Divided Brains: The Biology and Behaviour of Brain Asymmetries. Cambridge University Press, Cambridge. http://dx.doi.org/10.1017/CBO9780511793899

[7] Herbert, M.R., Ziegler, D.A., Deutsch, C.K., O’Brien, L.M., Kennedy, D.N., Filipek, P.A., Bakardjiev, A.I., Hodgson, J., Takeoka, M., Makris, N. and Caviness Jr., V.S. (2005) Brain Asymmetries in Autism and Developmental Language Disorder: A Nested Whole-Brain Analysis. Brain, 128, 213-226. http://dx.doi.org/10.1093/brain/awh330

[8] Mitchell, R.L. and Crow, T.J. (2005) Right Hemisphere Language Functions and Schizophrenia: The Forgotten Hemisphere? Brain, 128, 963-978. http://dx.doi.org/10.1093/brain/awh466

[9] Heim, S. and Keil, A. (2004) Large-Scale Neural Correlates of Developmental Dyslexia. European Child \& Adolescent Psychiatry, 13, 125-140. http://dx.doi.org/10.1007/s00787-004-0361-7

[10] Sato, Y., Mori, K., Koizumi, T., Minagawa-Kawai, Y., Tanak, A., Ozawa, E., Wakaba, Y. and Mazuka, R. (2011) Functional Lateralization of Speech Processing in Adults and Children Who Stutter. Frontiers in Psychology, 2, 1-10. http://dx.doi.org/10.3389/fpsyg.2011.00070

[11] Johnson, M.B., Kawasawa, Y.I., Mason, C.E., Krsnik, Z., Coppola, G., Bogdanovic, D., Geschwind, D.H., Mane, S.M., State, M.W. and Sestan, N. (2009) Functional and Evolutionary Insights into Human Brain Development through Global Transcriptome Analysis. Neuron, 62, 494-509. http://dx.doi.org/10.1016/j.neuron.2009.03.027

[12] Pascual, A., Huang, K.L., Nevue, J. and Préat, T. (2004) Brain Asymmetry and Long-Term Memory. Nature, 427, 605606. http://dx.doi.org/10.1038/427605a

[13] Skeath, J.B. and Thor, S. (2003) Genetic Control of Drosophila Nerve Cord Development. Current Opinion in Neurobiology, 13, 8-15. http://dx.doi.org/10.1016/S0959-4388(03)00007-2

[14] Certel, S.J. and Thor, S. (2004) Specification of Drosophila Motoneuron Identity by the Combinatorial Action of POU and LIM-HD Factors. Development, 131, 5429-5439. http://dx.doi.org/10.1242/dev.01418

[15] Guest, S.T., Yu, J.K., Liu, D.M., Hines, J.A., Kashat, M.A. and Finley, R.L. (2011) A Protein Network-Guided Screen for Cell Cycle Regulators in Drosophila. BMC Systems Biology, 5, 65. http://dx.doi.org/10.1186/1752-0509-5-65

[16] Bechtold-DallaPozza, S., Hiedl, S., Roeb, J., Lohse, P., Malik, R.E., Park, S., Durán-Prado, M. and Rhodes, S.J. (2012) A Recessive Mutation Resulting in a Disabling Amino Acid Substitution (T194R) in the LHX3 Homeodomain Causes Combined Pituitary Hormone Deficiency. Hormone Research in Paediatrics, 77, 41-51. http://dx.doi.org/10.1159/000335929

[17] Nakamura, M., Baldwin, D., Hannaford, S., Palka, J. and Montell, C. (2002) Defective Proboscis Extension Response 
(DPR), a Member of the Ig Superfamily Required for the Gustatory Response to Salt. The Journal of Neuroscience, 22, 3463-3472.

[18] Dubnau, J., Chiang, A.S., Grady, L., Barditch, J., Gossweiler, S., McNeil, J., Smith, P., Buldoc, F., Scott, R., Certa, U., Broger, C. and Tully, T. (2003) The Staufen/Pumilio Pathway Is Involved in Drosophila Long-Term Memory. Current Biology, 13, 286-296. http://dx.doi.org/10.1016/S0960-9822(03)00064-2

[19] Edwards, A.C., Zwarts, L., Yamamoto, A., Callaerts, P. and Mackay, T.F. (2009) Mutations in Many Genes Affect Aggressive Behavior in Drosophila melanogaster. BMC Biology, 7, 29. http://dx.doi.org/10.1186/1741-7007-7-29

[20] Blanco, E., Pignatelli, M., Beltran, S., Punset, A., Pérez-Lluch, S., Serras, F., Guigó, R. and Corominas, M. (2008) Conserved Chromosomal Clustering of Genes Governed by Chromatin Regulators in Drosophila. Genome Biology, 9, R134. http://dx.doi.org/10.1186/gb-2008-9-9-r134 